\title{
Design, Principles, and Testing of a Latching Modular Robot Connector
}

\author{
Nick Eckenstein and Mark Yim
}

\begin{abstract}
Connection and disconnection occur often in modular robotics. Furthermore, position errors increase in chain-style modular robots as chains get longer. This paper presents a passive (unactuated) compliant two-layer latching mechanism compatible with planar docking mechanisms such as the $\mathrm{X}$-Face, with design considerations and important design parameters empirically identified. Design parameters such as alignment, face curvature and overhang width are shown to have unintuitive effects on the behavior and strength of the connectors. The latch mechanism has a bonding ratio (force of the bond over force required to engage bond) in the range of 20 to 76, depending on the design parameters used. The paper also presents a reconfiguration control sequence that combines the module actuation forces with the connectors' natural forces to increase the reliability of the connection process and reduce the maximum force required. In addition, several types of reconfiguration are performed, including reconfiguration with a 12 module long chain that demonstrates the connectors' robustness to error.
\end{abstract}

\section{INTRODUCTION}

Mechanisms in the field of modular robotics have advanced with the development of many different systems by teams around the world. These systems have been classified into three areas: lattice denoting systems which nominally sit on a lattice and reconfigure by changing connections with neighbors, chain denoting systems which form and reform into chains, and mobile denoting systems which are capable of movement independent of other modules [1]. Reconfiguration is accomplished by repetition of a process known as docking and undocking, the physical connection and disconnection of two modules.

The docking process is divided into two parts: alignment, in which the modules obtain a sufficiently accurate relative position, and latching where the physical connection between the modules is made or forces put in place which maintain the alignment. Our previous work was focused on the alignment capabilities of 2D and 3D connection mechanisms, dubbed 'X-Face' and 'X-Face 3D' [2] [3]. In this work, we attempt to design a suitable latching mechanism that works with maximum alignment (X-Face) shapes in $2 \mathrm{D}$, as well as determine important design parameters and control sequence.

In general, docking between two modules occurs at special docking faces on a module called connectors. Faces can be gendered or ungendered. Gendered faces have male and female features which must be matched, and thus must be carefully controlled to ensure connections are formed from one of each face. Ungendered faces do not have this restriction. Hermaphroditic faces have both male and female features on each face.

Another requirement for reconfigurable connectors is the ability to undock successfully as well as dock. If mechanical

Nick Eckenstein and Mark Yim, GRASP Lab and Department of Mechanical Engineering and Applied Mechanics, at University of Pennsylvania, email: neck@ seas.upenn.edu. connection is being used, either the docking or undocking requires some actuation capabilities. One or the other of these processes can proceed passively but in order for the connection to be stable under all possible force/torque conditions, there needs to be an active state change. Historically this has been accomplished by SMA or small motors [4] [5].

In our previous work on the 2D X-Face, we introduced the term area of acceptance, which was defined as "the range of possible starting conditions for which mating will be successful". Simply speaking, it represents the possible position and orientation offsets from ideal conditions which will still result in the alignment of the two faces. This quantity varies between connectors and is used as a metric of comparison.

\section{A. Related Work}

A wide variety of specialized connectors for modular robots exists in the literature, typically magnetic, electrostatic, or mechanical [3]. The hermaphroditic DRAGON Connector has a high area of acceptance classified in several individual dimensions of offset (linear and angular), and contains a heavy-duty passive latching mechanism to withstand high loads [6]. The SINGO Connector is a hermaphroditic connector with an actively driven spiral gear mechanism that ultimately results in high error correctability over certain directions of offset [7]. The hermaphroditic X-Claw connector [8] uses an active gripper and mechanical self-alignment features to ensure a high area of acceptance as well as 4 possible attachment orientations between two given faces. Unfortunately the X-Claw also suffered from a relatively loose grip and weak component strength.

Docking connectors have also been used and studied in aerospace applications (i.e. rendezvous and docking systems), however the essential requirements and mission can be very different and thus result in different systems. Space docking mechanism requirements include robustness, impact absorption, and reversibility [9]. Modular robots connector design on the other hand prioritizes tolerance to alignment errors (area of acceptance), small profile size, and low power and actuation [1]. These elements lead us to study passive mechanical self-aligning mechanisms with high area of acceptance as the basic shape for our designs.

\section{LATCHING X-FACE DESIGN}

Previously we presented the X-Face [2], a 2D connector made from two layers of material (making it more like 2.5D) which we showed in simulation to have a significantly larger area of acceptance than shapes proposed by Nilsson [10] which are considered to be the upper bound for planar connectors. Expanding on the concepts from the 2D X-Face, we present a new latching design for connection of modular robots which we simply call the 'Latching X-Face'. 

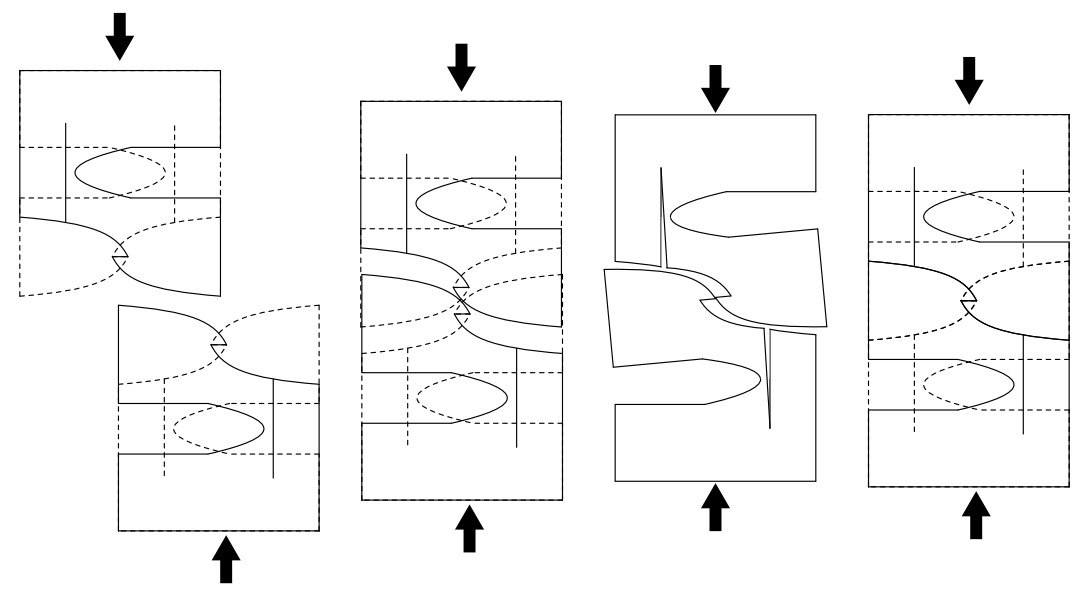

Fig. 1: Connection sequence. Top layer-solid, bottom layer-dashed. Bottom layer is removed for clarity in the 3rd image.

The design goals for this new connector include: a latching functionality, low required force to actuate, and high strength when latched. To that end, the connector has no actuated components per se; energy for the latching operation is given by the motion of the modules that hold it. Likewise, we hope to keep the force required for latching low while retaining high bonding strength. To that end we will use as a performance metric, the bonding ratio, which is the force required to disconnect (in this case, material failure) divided by the force required to connect. This is used to give us one performance metric for our connector design.

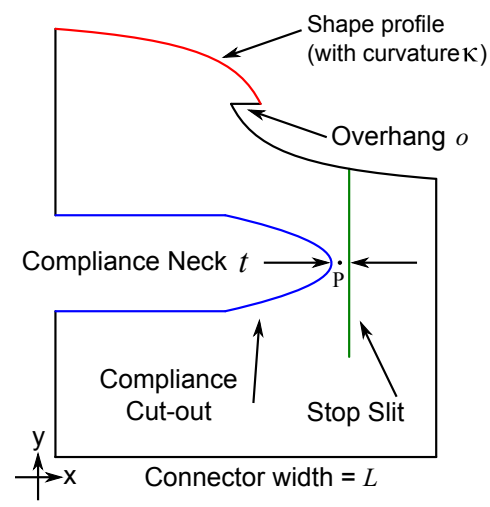

Fig. 2: Latching X-Face with important design features.

As in the 2D X-Face, two layers are attached to a face of a module and then moved by the modules to be adjacent to a target module, forming a connection. One layer is a mirror image of the other. The docking process for this design can be seen in Fig. 1 and follows:

1) Modules move in the plane to the target locations.

2) Connectors align by combination of module actuation forces and the mechanical connector interaction forces.

3) Modules are pressed closer together, forcing the compliant features outward.

4) Compliant features snap into the latched position to hold the docking connection together.

When the connector is used on a chain of cube or squareshaped modules such as CKBot [11] that is moving in a plane, we might wish to have connectivity to neighboring modules in all four adjacent locations (front, back, left, and right). However, in an articulated chain, at least one degree of freedom is required between two faces. Rigidly connecting all four sides would prevent this. Therefore, for our planar connector prototype we use three latching faces and a profile swept out to allow relative motion.

One layer of one side of the design is shown, with important components labeled, in Fig. 2. These layers are rigidly attached to each other and the module below (in the y direction) the compliant features so that they still function as intended. This combination of features results in a hermaphroditic connector which creates a strong mechanically latched connection without requiring actuators, but still has a high area of acceptance.

Prototypes can be seen in Fig. 3. The prototypes have a small separator layer between the X-Face layers to prevent interactions between those layers due to small deformations or misalignments. If the separator layer is equal to or greater than the width of the X-Face layers, the connectors can easily be disconnected manually by displacing them in the out-ofplane direction. The separator layer also prevents incidental interaction between layers due to small manufacturing defects or bending. We used 1/4" ABS lasercut plastic for all three layers on the prototypes. Prototypes for bonding ratio testing have one latching face while those used for reconfiguration demonstrations have three latching faces. These will be referred to as the 'one-sided' and 'three-sided' prototypes, respectively. We elected to build the prototypes at a scale on par with CKBot, our target platform [11]. CKBot modules measure $65.5 \mathrm{~mm} \times 65.5 \mathrm{~mm} \times 90.4 \mathrm{~mm}$. The distance between rotors in a chain is $95 \mathrm{~mm}$, setting the center-tocenter distance between the connectors.

Therefore on the three-sided prototype (Fig. 3b), the distance from the center of the overhang to the center of the shape which is placed over the rotor is $47.5 \mathrm{~mm}$, with the width of a latching face for the connector, $65.5 \mathrm{~mm}$.

\section{Design Principles And Parameters}

There are several qualitative design principles and corresponding quantitative design parameters which are essential 


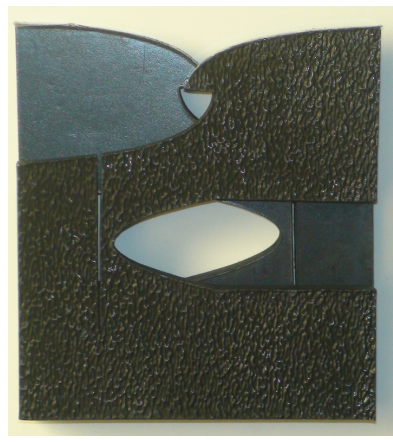

(a) Single sided prototype

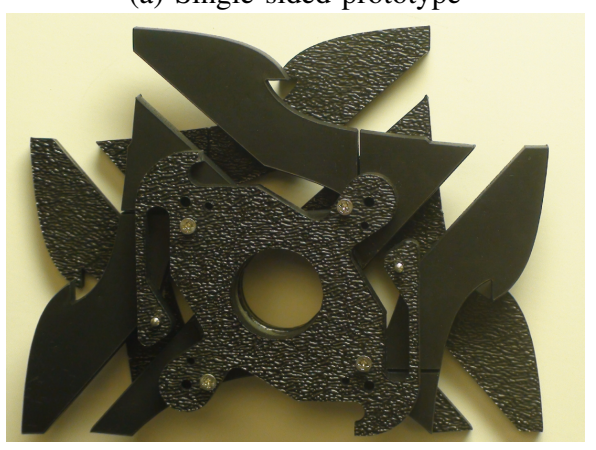

(b) Three-sided prototype. ModLock [11] (shown on top) connects to CKBots.

Fig. 3: Latching X-Face prototypes, close view.

to the function of this latching mechanism (refer to the labeled components of Fig. 2).

Profile Maximum. Like the 2D X-Face, the highest point on the shape profile is at the outer edge. If we let the full width of the connector be $L$, and the width of the overhang be $o$, we can view the shape profile as a function $\left(f(x) \mid x \in\left[0, \frac{L}{2}+\frac{o}{2}\right]\right)$. Then the function is at its maximum at $f(0)$. Furthermore, the profile function should be decreasing at all points: $f^{\prime}(x)<0 \forall x \in\left[0, \frac{L}{2}+\frac{o}{2}\right]$ to prevent jamming. The two layers permit full alignment in either direction. These characteristics ensure high area of acceptance, similar to the 2D X-Face. Note that the shape profile is rotationally symmetric about the overhang, so the male and female features of mating faces nest properly.

Overhang. Represented by the value $o$, the overhang prevents the connector from becoming disconnected up to material failure; with the previous X-Face design, detachment was not prevented by separating motions along the mating axis. The width of the overhang determines how much deformation of the connector is necessary to perform latching, and thus affects the maximum force required.

Compliance. The shape and location of the compliant cutout as well as the distance from the stop slit (which we designate $t$ ) determines the compliance. This distance $t$, is the thickness at the narrowest point of the section, which we call the compliance neck.

Curvature. We found that if the curvature $\kappa$ is concave (which we will call positive curvature) or straight, the connectors had a tendency to jam.This was due to an interaction with the bent position of the latch arm with the shape. Once the arm begins to bend, forces change their direction and begin pointing away from the inwards alignment direction provided the curvature is non-negative. If the curvature is negative the rotation due to bending has a reduced effect on the direction of the interaction forces, ensuring a good alignment.

Mechanical Stop. Not only does the stop slit allow rotation of the necessary features, it prevents undesired rotation in the opposite direction. Without the mechanical stop section of the connector, the compliance would work in both directions, preventing rigidity under tension. The relevant quantities for the stop slit are the depth (y distance down from the curved face) and width (gap size in $\mathrm{x}$ ). Width should be small to keep the connector aligned under tension, while the depth is ideally lower than the closest point on the compliance cutout to allow good compliance. The stop slit itself is located with sufficient $\mathrm{x}$ distance from the rightmost end to ensure sufficient rigidity under tension. A distance of $15 \mathrm{~mm}$ was found to be sufficient. A depth of $20.3 \mathrm{~mm}$ was found to be sufficient for the compliance to work correctly. The gap for the stop is equal to two times the laser cut kerf, or $0.36 \mathrm{~mm}$.

We model the compliant section by a pseudo-rigid body model (PRBM) [12]. This method equates the compliant mechanism to two rigid bodies connected by a torsional spring with an equivalent spring stiffness, and for simple cantilevers predicts the deflection path accurately within 0.5 percent [13]. The torsional stiffness for a simple PRBM model of this type is $k=\frac{E I}{l_{e q}}$ where $E$ is the Young's Modulus of the material (our prototypes use ABS, $E=2.1 \mathrm{GPa}$ ), $I$ is the area moment of inertia of the cross-section (assumed to be equivalent to a thin rectangular beam), and $l_{e q}$ is the equivalent length. This model can be used to scale the system or adapt to changes in material properties and thickness. The equivalent stiffness for our three-sided prototype is approx. $4.99 \mathrm{Nm} / \mathrm{rad}$. Since the corresponding relationship between deflection and moment is $M=k \theta$, overhang increases result in an increase in the maximum moment (thus force) required. The compliance neck thickness $t$ affects directly the area moment of inertia $I$, as does the material thickness.

\section{Force Testing}

In order to accurately measure the bonding forces of the connector, testing was performed using an MTS Criterion $^{\mathrm{TM}}$ Model 43 Testing System. Single-sided prototypes (including both layers) were mounted to a central piece which was then clamped into place on machine base and crosshead, respectively. Two types of experiments were performed; compression to determine the force required to perform latching, and latched extension until material failure to determine the load strength. Two typical tests are shown in Fig. 4. The latching for each of the two layers is visible in Fig. 4a. When a layer latches, a sudden dip in force can be seen. In this test the two layers latch one at a time allowing us to see both latchings at approximately 46 and 48 seconds. Force then quickly increases again as the assembly is compressed.

In order to explore the design space, prototypes were created that varied parameters described in Section III.

The profile maximum was kept at $x=0$ primarily due to the $2 \mathrm{D} \mathrm{X}$-Face, which relies mostly on this feature for 


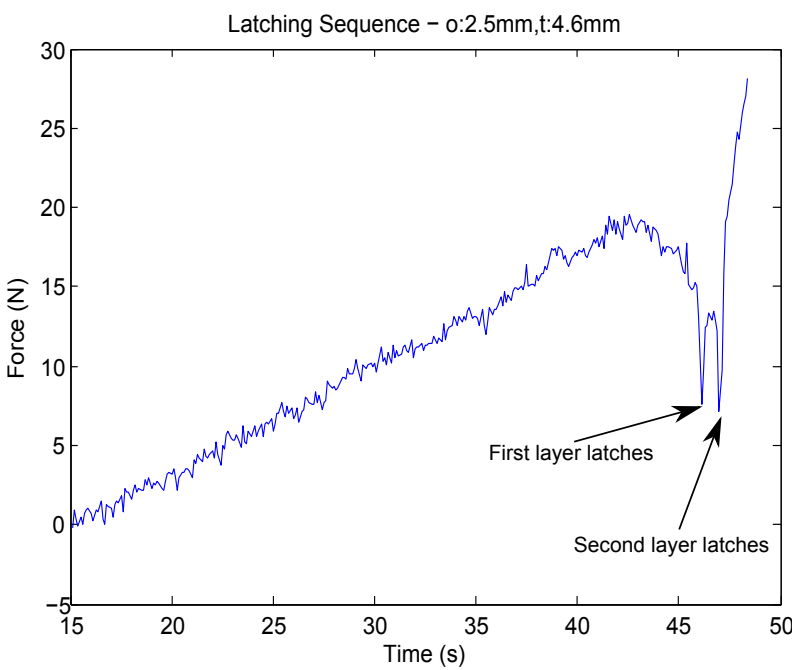

(a) Compression test showing typical test and noise characteristics. Successful latching of each of the two layers is visible.

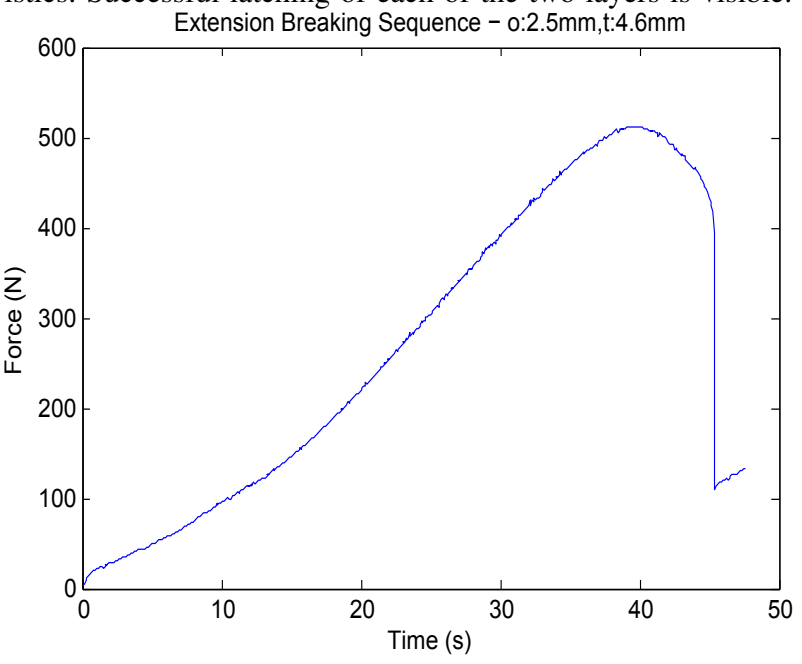

(b) Tension break test: Deformation is visible before failure.

Fig. 4: Force test plots

high area of acceptance. Prototypes were observed to have no difficulty correcting errors over approximately the same range of linear errors as the $2 \mathrm{D} \mathrm{X}$-Face when the one-sided prototype was connected by hand.

The final design curvature was defined by an interpolated spline. The outermost end of the spline (at $x=0$ ) was set to an angle of $35^{0}$ with the x-axis seen in Fig. 2. Smaller angles tended to deflect without alignment; this counterproductively changes the direction of the force vector. Larger angles create too flat a surface, increasing the surface area of friction and thus the force required to align and latch.

To increase the torque applied throughout the curvature, the spline at the end touching the overhang was defined so as to 'point' directly to the rotation point of the compliant section. In other words, if the spline was extended in a straight line from the overhang at the same angle it would intersect the midpoint of the compliance neck, labeled $\mathrm{P}$ in Fig. 2. This point is assumed to be the center of rotation for the flexure above based on observation of the flexing behavior. Other forces are approximately tangent to that line.

Since they both effect the force required for latching and thus the bonding ratio, six different connector designs with varying $o$ and $t$, were tested. Results including compressive/extensive forces and spring constants as well as the bonding ratio can be seen in Table I. We expect increases in $o$ and $t$ measurements to increase the forces required to connect. Increasing $o$ requires increased deflection. Increasing compliance neck thickness $t$ gives us a stiffer member. Both of these would result in larger forces. Prototypes with an overhang $o$ of $1.27 \mathrm{~mm}$ were constructed, but found to be too difficult to get a good latch - often the overhang would overlap less than $1 \mathrm{~mm}$, resulting in unstable connections. These prototypes were therefore excluded from testing.

The mechanical stop features were found to be sufficient to allow for compliance and rigidity, and not examined.

Results in the table are somewhat unintuitive, but testing observations reveal more information. Designs with the smaller overhang $(o<=2.5 \mathrm{~mm})$ and a large neck $(t=3.0$ or $t=4.6 \mathrm{~mm}$ ) were observed to have a different failure mechanism from the other connector prototypes. Most of the prototypes failed at the narrow band between the compliance cut-out and the stop slit (the compliance neck), breaking into two large pieces. The prototypes with $o=2.5 \mathrm{~mm}$ and $t=3.0 \mathrm{~mm}$ or greater failed at the tip of the overhang, deforming the material until the parts slipped apart. This change in failure characteristic is responsible for the seeming discrepancies in the Extension Force numbers. Note that the extension spring constant $k_{E}$ increases even when the maximum force decreases for the third prototype. The change in failure characteristic causes failure at less force and distance, effectively weakening the material without effecting the spring constants.

Due to the change of the mode of failure, the effect of the compliance neck thickness $t$ changed. With failure at the neck, a thicker neck predictably improved the strength of the connector. However, with failure at the overhang, a thinner (i.e. more compliant) neck improved the strength.

Greater compliance leads to greater deflection for a given force as expected. Deflection of the connector (now clockwise about $\mathrm{P}$ ) helps prevent deformation at the overhang tip by changing the angle of the overhang relative to the force, allowing a stronger portion of the layer to take on the extension forces. The deflection also pushes the tips of the overhangs into the corresponding trench on the opposing connector, effectively acting like reciprocal hooks. When the overhang is held more rigidly (less compliance), the tips deform more easily as they share a greater portion of the force.

Otherwise, the data fits expectations - greater thicknesses increase both the force required to latch and the force required to break by extension. Effective spring constants increased with increased thickness as well. Interestingly, the bonding ratio decreased as the material got thicker - the force to connect increased faster than the force to break.

For the three-sided prototype some alterations to the compliance cut-out were necessary to fit the $90^{\circ}$ profile as seen in Fig. 3b. These include narrowing the width of the compliance cut-out and changing the angle to fit the shape. As a result, the shape is slightly more compliant than would be expected of the same $o$ and $t$ measurements tested. So 
TABLE OF STRESS TESTING RESULTS

\begin{tabular}{|c|c||c|c|c|c|c|}
\hline$o(\mathrm{~mm})$ & $t(\mathrm{~mm})$ & Compression Force - Latching $(\mathrm{N})$ & Extension Force - Failure $(\mathrm{N})$ & $k_{C}(\mathrm{~N} / \mathrm{mm})$ & $k_{E}(\mathrm{~N} / \mathrm{mm})$ & Bonding ratio \\
\hline 2.5 & 2.3 & 6.18 & 489.11 & 9.674 & 84.76 & 79.1 \\
2.5 & 3.0 & 8.56 & 666.39 & 44.19 & 92.63 & 77.8 \\
2.5 & 3.8 & 10.42 & 669.48 & 27.58 & 125.7 & 64.2 \\
2.5 & 4.6 & 19.57 & 512.32 & 69.37 & 141.8 & 26.2 \\
3.8 & 3.8 & 14.55 & 824.03 & 25.20 & 147.8 & 56.6 \\
5.1 & 2.3 & 9.32 & 508.77 & 7.526 & 86.02 & 54.6 \\
5.1 & 3.0 & 16.36 & 646.74 & 26.01 & 108.0 & 39.5 \\
5.1 & 3.8 & 22.34 & 813.09 & 30.78 & 149.2 & 36.4 \\
5.1 & 4.6 & 46.58 & 960.10 & 91.22 & 134.6 & 20.6 \\
\hline
\end{tabular}

TABLE I: Varied Parameters Force Testing Results. Forces shown are the highest recorded during the relevant activity, which may be $\pm 1 \mathrm{~N}$. $k_{C}$ and $k_{E}$ are the spring constants in compression and extension respectively, starting from zero deflection.

despite using the parameters $o=5.1 \mathrm{~mm}$ and $t=3.0 \mathrm{~mm}$, this connector requires $9.61 \mathrm{~N}$ to latch and $584 \mathrm{~N}$ to disconnect, giving a bonding ratio of 60.9:1.

\section{RECONFIGURATION TESTING}

Verification of assembly as part of reconfiguration started from a preassembled planar chain of CKBot [11] modules. The three-sided version of the Latching X-Face Connector attached to one face of each module, with casters below to reduce friction.

Three different reconfiguration sequences were tested and are shown in Fig. 5. The first example simulates a single lattice-type operation, which we call the ' $\mathrm{P}$ ' shape. The second, called the 'Block' (essentially a $3 \times 2$ cluster of modules) starts with a straight $1 \times 6$ preassembled chain using ModLock connectors. It demonstrates a continuation of the ' $\mathrm{P}$ ' shape lattice style reconfiguration; showing progressive latching (one module after another). This also demonstrates the capability to perform latching on mid-chain modules. Inductively, we assume more modules are capable of as much latching as required, done sequentially in a zipper-like fashion.

The third shape, a large square demonstrates chain style reconfiguration. This 12-module chain was assembled similarly to the others, but with connectors only at the chosen docking site. This is an important configuration because long chains of modules accumulate positional errors requiring large area of acceptance. When performing the attachment, we select kinematic arrangement that allows a large application of torque at the connection site.

The attachment process itself is a series of open-loop position commands. This command sequence requires some tuning to ensure a good latching connection, but once tuned correctly, completed 6 trials for each reconfiguration with $100 \%$ reliability.

The CKBot system uses EX-106+ Dynamixel Servos. with a stall torque of $10.9 \mathrm{Nm}$. The reconfiguration command sequences were optimized for speed; as such the actual application of force is not defined. However, our data has shown that any system which can provide a sustained force over $9.61 \mathrm{~N}$ should be able to successfully latch.

\section{A. Latching Sequence}

The latching sequence includes a set of positional commands to the servos as shown in Tab. II. The servos use position control so while performing reconfiguration testing, commands go slightly beyond the nominal position of latching in order to apply appropriate force to engage the compliant members. For the two 6-module reconfiguration sequences, we refer to the modules in positional order starting from the closest to the control computer (i.e. module 1 is closest, module 6 is furthest).

In Tab. II, for the single attachment in the ' $\mathrm{P}$ ' shape reconfiguration, the first step of module 4 (at second 1) converts the chain to an L-shape that is slightly less than $\frac{\pi}{2}$. This means the connector will be slightly off-center in the next step at second 3 - the connector then only requires force to overcome the latch on a single layer, rather than both if it were centered. Once the first layer is latched by driving module 5 well past $-\frac{\pi}{2}$ at second 3 , both modules are commanded to 'go slack', that is, apply no force. This allows the connectors to realign from the structural forces in the connector flexure. Before this slack motion, the two connectors are not aligned. Module 5 is then commanded past $-\frac{\pi}{2}$ once more to ensure the second layer latches. The configuration then goes slack once more to realign the faces.

A plot of the motions as sensed by the encoders in modules 4 and 5 is shown in Fig. 6. At second 3, the module moves past the nominal $-\frac{\pi}{2}$ corresponding to the latch of a single layer. At second 4, both modules go slack, allowing the connector forces to take over and realign both modules closer to the $-\frac{\pi}{2}$ position. Finally at second 5 , module 5 uses the second motion command that completes the final latch. Second 6 makes both modules slack, to draw no power. The same sequence was repeated for the relevant modules in the 'Block' shape reconfiguration, and a similar procedure for the chain reconfiguration.

CONTROL SEQUENCE TABLE

\begin{tabular}{|c|c|c|}
\hline Time(s) & M4 Pos. Comm. (rad) & M5 Pos. Comm. (rad) \\
\hline 0 & 0 & 0 \\
1 & -1.67 & 0 \\
3 & -1.67 & -1.74 \\
4 & GS & GS \\
5 & GS & -1.74 \\
6 & GS & GS \\
\hline
\end{tabular}

TABLE II: Control sequence for latching. GS stands for the 'go slack' command.

\section{CONCLUSiOn AND Future Work}

We have presented a new connector design for a 2D modular robot that includes high self-alignment and reliable latching. Design principles used to create the connector are presented and explained, along with the corresponding quantitative measurements. Force testing to determine the impact of design parameters was presented, along with analysis of the corresponding behaviors. Bonding ratios were obtained 

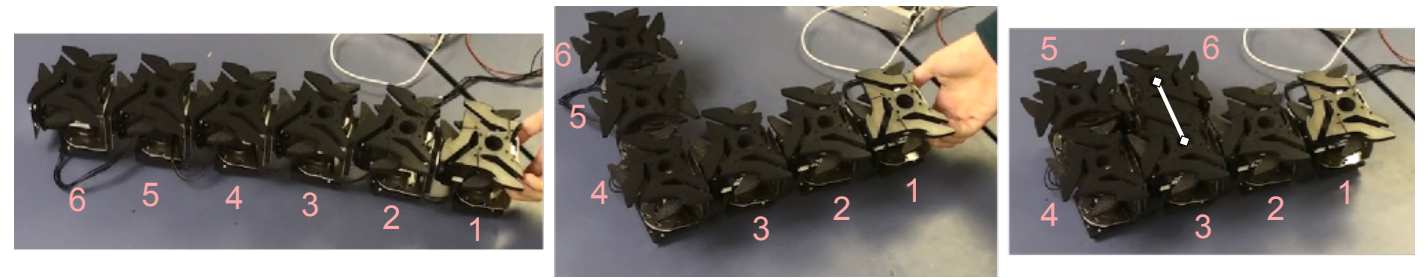

(a) P-shape (basic) reconfiguration
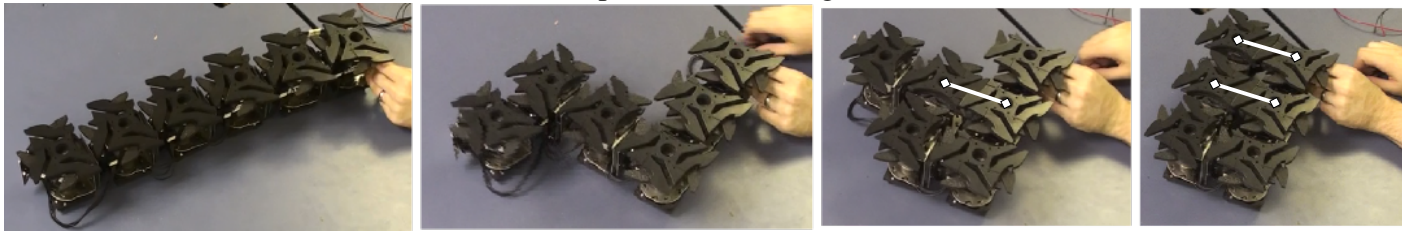

(b) Block-shape (lattice style) reconfiguration
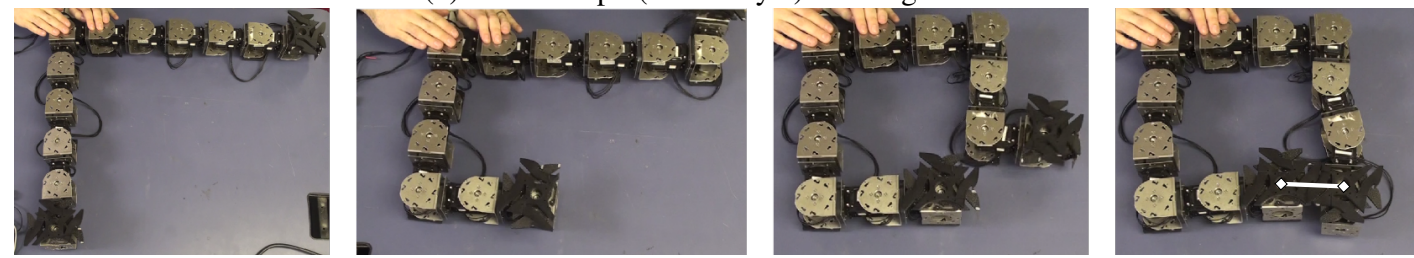

(c) O-shape (chain style) reconfiguration

Fig. 5: Reconfiguration sequences detail. Diamond-end white lines indicate latch connection sites.

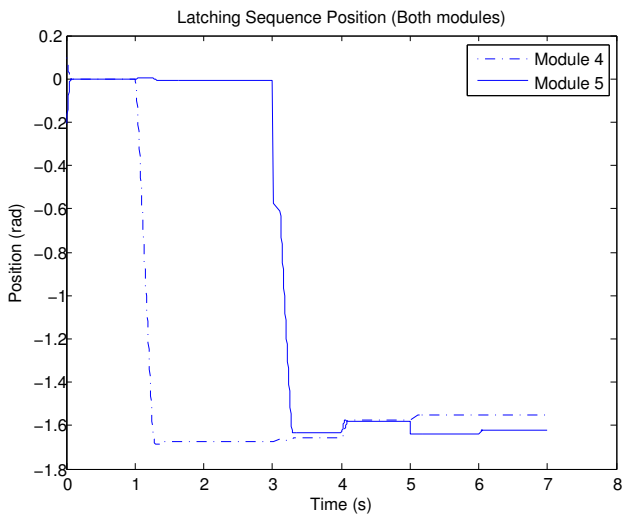

Fig. 6: Latching sequence positions of modules 4 and 5.

for connector prototypes in the range from 20.6 to 79.1 . While ABS plastic was the only material tested, different materials such as metals with sufficient elastic regions may yield much higher bonding ratio.

Reconfiguration on a 2D CKBot chain was performed and demonstrated to show simple attachment as well as progressive attachment of multiple connectors. Reconfiguration also demonstrated capabilities for lattice-style as well as chain-style attachment. Finally, the latching control sequence taking advantage of the natural forces present in the connector was presented.

In the future, we will seek to make the connector capable of controlled disconnection. This will require pulling the compliant lever arms down by use of some actuator. SMA wires would provide a low-profile and low-cost solution, although they may be slow. Mechanically similar systems have been used to bend compliant arms with SMA [14], [5].

\section{REFERENCES}

[1] K. Stoy, An Introduction to Self-Reconfigurable Robots. Boston, MA: MIT Press, 2009.

[2] N. Eckenstein and M. Yim, "The x-face: An improved planar passive mechanical connector for modular self-reconfigurable robots," in Intl. Conf. on Intelligent Robots and Systems (IROS). IEEE/RSJ, 2012, pp. 3073-3078.

[3] — "Area of acceptance for 3d self-aligning robotic connectors: Concepts, metrics, and designs," in ICRA . Proc. of the IEEE Intl. Conf. on Robotics and Automation,. IEEE, 2014.

[4] H. Kurokawa, K. Tomita, A. Kamimura, S. Kokaji, T. Hasuo, and S. Murata, "Distributed self-reconfiguration of m-tran iii modular robotic system," vol. 27, no. 3-4, pp. 373-386, 2008.

[5] M. Yim, Y. Zhang, K. Roufas, D. Duff, and C. Eldershaw, "Connecting and disconnecting for chain self-reconfiguration with polybot," Mechatronics, IEEE/ASME Trans. on, vol. 7, no. 4, pp. 442-451, 2002.

[6] M. Nilsson, "Connectors for self-reconfiguring robots," IEEE/ASME Trans. on Mechatronics, vol. 7, no. 4, pp. 473-474, 2002.

[7] W.-M. Shen, R. Kovac, and M. Rubenstein, "Singo: A singleend-operative and genderless connector for self-reconfiguration, selfassembly and self-healing," in ICRA. IEEE Intl. Conf. on Robotics and Automation, 2009, pp. 4253-4258.

[8] J. J. Cong and R. Fitch, "The x-claw self-aligning connector for selfreconfiguring modular robots," in IROS '11: Proc. of the Workshop on Reconfigurable Modular Robotics: Challenges of Mechatronic and Bio-Chemo-Hybrid Systems, 2011.

[9] F. Gampe, K. Priesett, and R. Bentall, "A modular docking mechanism for in-orbit assembly and spacecraft servicing," in 19th Aerospace Mechanisms Symposium 1985, 1985, pp. 59-74.

[10] M. Nilsson, "Symmetric docking in 2d: A bound on self-alignable offsets," in IASTED '99: Robotics and Automation, Oct. 1999.

[11] J. Davey, J. Sastra, M. Piccoli, and M. Yim, "Modlock: A manual connector for reconfigurable modular robots," in IEEE/RSJ Intl. Conf. on Intelligent Robots and Systems (IROS), 2012, pp. 3217-3222.

[12] L. L. Howell and A. Midha, "A method for the design of compliant mechanisms with small-length flexural pivots," J. of Mechanical Design, vol. 116, no. 1, pp. 280-290, 1994.

[13] L. Howell, A. Midha, and T. Norton, "Evaluation of equivalent spring stiffness for use in a pseudo-rigid-body model of large-deflection compliant mechanisms," J. of Mechanical Design, vol. 118, no. 1, pp. 126-131, 1996.

[14] P. J. White and M. Yim, "Reliable external actuation for full reachability in robotic modular self-reconfiguration," The Intl. J. of Robotics Research, vol. 29, no. 5, pp. 598-612, 2010. 